\title{
Obesity and Race May Explain Differential Burden of White Matter Hyperintensity Load
}

\author{
Azizi A Seixas $\mathbb{D}^{1,2}$ \\ Arlener D Turner (D) ${ }^{2}$ \\ Omonigho Michael Bubu',2 \\ Girardin Jean-Louis (D) ${ }^{1,2}$ \\ Mony J de Leon ${ }^{3}$ \\ Ricardo S Osorio ${ }^{2}$ \\ Lidia Glodzik ${ }^{3}$
}

'New York University Grossman School of Medicine, Department of Population Health, New York, NY, I00I6, USA;

${ }^{2}$ New York University Grossman School of Medicine, Department of Psychiatry, New York, NY, I0016, USA; ${ }^{3}$ Weill Cornell Medicine, Department of Radiology, New York, NY, I002I, USA
Correspondence: Azizi A Seixas Departments of Population Health and Psychiatry, New York University Grossman School of Medicine, 180 Madison Avenue, Office \# 715, New York, NY, 10016, USA

Tel + I 646-50I-3430

$\mathrm{Fax}+1$ 2/2-263-4595

Email Azizi.Seixas@nyulangone.org
Objective: Compared to European Americans, research indicates that African Americans have higher white matter hyperintensity (WMH) load; however, the clinical and biological bases underlying this higher burden are poorly understood. We hypothesize that obesity may explain differences in WMH between African and European Americans.

Methods: Participants enrolled in longitudinal brain aging studies ( $\mathrm{n}=292 ; 61 \%$ Female; 92\% European American; mean age $=69.6 \pm 7.7$ ) completed evaluations including medical exams, neuroimaging, and sociodemographic surveys. Overweight/obese status defined as body mass index $\geq 30 \mathrm{~kg} / \mathrm{m}^{2}$, and WMH load, captured by FLAIR images, as sum of deep and periventricular volumes, scored using the Fazekas scale (0-6), WMH $\geq 4$ considered high. Results: Logistic regression analyses, adjusted for age, sex, hypertension, and smoking history, indicated that age and interaction between race and obesity were significant predictors of WMH, demonstrating that obesity significantly moderated the relationship between race and WMH. Age independently increased the odds of high WMH by $16 \%(\mathrm{OR}=1.16$, $95 \% \mathrm{CI}=1.09-1.23, \mathrm{p}<0.001)$. Stratified analysis indicates that older European Americans had increased WMH $(\mathrm{OR}=1.17,95 \% \mathrm{CI}=1.09-1.23, \mathrm{p}<0.001)$, while obese African Americans had increased WMH $(\mathrm{OR}=27.65,95 \% \mathrm{CI}=1.47-519.13, \mathrm{p}<0.05)$. In a case controlled subgroup matched by age, sex, and education $(n=48)$, African Americans had significantly higher WMH load $\left(27 \%\right.$ vs $\left.4 \%, X^{2}=5.3, \mathrm{p}=0.02\right)$.

Conclusion: Results denote that age predicted WMH among European Americans, while obesity predicted WMH among African Americans. Matched sample analyses indicate that obesity increases the odds of WMH, though more pronounced in African Americans. These findings suggest that obesity may explain the differential burden of white matter hyperintensity load, signifying public health and clinical importance.

Keywords: white matter hyperintensities, WMH, racial/ethnic differences, African American, obesity

\section{Introduction}

Although dementia and stroke affect all racial/ethnic groups, African Americans are disproportionately burdened compared to European Americans, ${ }^{1-4}$ a disparity that persists despite attempts to understand and reduce racial/ethnic differences through prevention programs. $^{4-6}$

White matter hyperintensities (WMH) are markers of cerebral small vessel disease - a cluster of subcortical lacunar infarcts (clinically symptomatic), white matter MRI hyperintensities, lacunes (clinically silent), prominent perivascular spaces, cerebral microbleeds, and atrophy $^{7}$ (highly prevalent in African Americans) $)^{8,9}$ and significant indicators of cognitive impairment, future stroke 
(hazard ratio (HR):3.3), dementia (HR:1.9), and death (HR:2.0). ${ }^{10}$ In epidemiological data, African Americans have a higher prevalence, ${ }^{11,12}$ higher overall volumes, and higher substantial progression rates of $\mathrm{WMH}$, regardless of age. ${ }^{11,13-15}$ Although previously, attributed to smoking history and hypertension, ${ }^{11,13}$ a seminal study in older adults with a family history of cardiovascular disease (CVD) and coronary artery disease found African Americans had $80 \%$ higher odds of severe WMH than European Americans, independent of age, sex, hypertension, and other risk factors. ${ }^{12}$

Though higher WMH burden may explain racial/ethnic differences in dementia, stroke, subcortical lacunes, ${ }^{16,17}$ and CVD-related mortality and provide a novel intervention target to prevent or reduce these differences, ${ }^{11,14}$ it is unclear what clinical and biological reasons underlie African Americans having a greater risk and higher burden of WMH. Further, evidence from clinical trials demonstrated that management of CVD risk via physical activity and diet lead to significantly lower WMH volume in overweight/obese individuals. ${ }^{18}$

To our knowledge, no examinations posit obesity as a primary contributor to racial/ethnic differences in WMH via matched sample. Therefore, we investigated through case control analysis whether obesity explained racial/ethnic differences in WMH between age, gender and education matched African and European Americans.

\section{Methods}

\section{Participants}

We identified a cohort of 308 participants, of which 292 had complete data for all variables. As such, the analyses were conducted on 292 older adults without dementia (61\% women; 92\% European American). The mean age of the sample was $69 \pm 7.7$ years $(\mathrm{EA}=70$ and $\mathrm{AA}=65.1)$ and had on average $16.6 \pm 2.3$ years of education $(\mathrm{EA}=16.7$ and $\mathrm{AA}=15.3$ ) (See Table 1). All participants were volunteers recruited at the NYU Center for Brain Health for longitudinal studies on brain aging and early diagnosis of Alzheimer's disease. All participants provided informed consent through signed IRB-approved consent forms and underwent medical examination), psychiatric, and neurological assessments, blood tests, electrocardiogram (ECG), and magnetic resonance imaging (MRI). Exclusion criteria included active or lifelong major depression (score $>16$ on 17-item Hamilton Depression Scale), ${ }^{19}$ brain tumor, neocortical infarction, Axis I psychiatric/
Table I Prevalence of WMH, Comorbidities, and Demographics by Race

\begin{tabular}{|l|l|l|l|}
\hline Variable & $\begin{array}{l}\text { European } \\
\text { Americans }\end{array}$ & $\begin{array}{l}\text { African } \\
\text { Americans }\end{array}$ & Sig \\
\hline Any PWMH (Score I-3) & $80.2 \%$ & $83.3 \%$ & 0.48 \\
Any DWMH (Score I-3) & $69.0 \%$ & $79.2 \%$ & 0.21 \\
Any WMH Score (I-6) & $86.9 \%$ & $95.8 \%$ & 0.17 \\
Severe PWMH (Score $\geq 2)$ & $26.9 \%$ & $37.5 \%$ & 0.19 \\
Severe DWMH (Score $\geq 2)$ & $17.9 \%$ & $20.8 \%$ & 0.45 \\
Severe WMH (Score $\geq 4)$ & $16.0 \%$ & $20.8 \%$ & 0.36 \\
Obesity & $22.0 \%$ & $29.0 \%$ & 0.28 \\
Hypertension & $41.8 \%$ & $41.7 \%$ & 0.58 \\
Smoking & $6.3 \%$ & $20.8 \%$ & $<0.05$ \\
Age (years) & $70.0 \pm 7.5$ & $65.1 \pm 8.2$ & 0.003 \\
Sex (\% women) & $59.7 \%$ & $70.8 \%$ & 0.20 \\
Education (years) & $16.7 \pm 2.3$ & $15.3 \pm 2.4$ & 0.002 \\
SBP (mmHg) & $122.4 \pm 15.3$ & $122.8 \pm 16.0$ & 0.83 \\
DBP (mmHg) & $71.8 \pm 10.0$ & $74.1 \pm 9.7$ & 0.27 \\
BMI & $26.4 \pm 5.2$ & $27.3 \pm 5.6$ & 0.58 \\
\hline
\end{tabular}

Notes: Age was compared using $t$-test, education, SBP, DBP, BMI using $U$ Mann Whitney test. Sample size: $n=292$ (268 European Americans and 24 African Americans).

Abbreviations: WMH, white matter hyperintensities; PWMH, presence of any periventricular white matter hyperintensities; DWMH, presence of any deep white matter hyperintensities; Severe WMH, presence of high white matter hyperintensity load; Obesity, body mass index $\geq 30 \mathrm{~kg} / \mathrm{m}^{2}$; Hypertension, diagnosed with hypertension disease; Smoking, current smoker; SBP, systolic blood pressure; DBP, diastolic blood pressure; BMI, body mass index.

mental health disorders, dementia, using the Clinical Dementia Rating (CDR) Scale and Mini Mental State Examination (MMSE) scores $<26 .{ }^{20}$ A study physician ruled out dementia based on a semi-structured diagnostic interview in addition to CDR and MMSE scores.

\section{Standard Protocol Approvals, Registrations, and Patient Consents}

The NYU Grossman School of Medicine Institutional Review Board approved all procedures performed in this study involving human participants. All study procedures were completed in accordance with the ethical standards of the NYU Grossman School of Medicine Institutional Review Board and with the Helsinki declaration and its amendments and written informed consent was obtained from all participants in the study.

\section{Clinical Measures}

Lab tests (in a fasting state) included complete blood count, metabolic and lipid panel, liver function tests and urinalysis. Obesity was calculated using body mass index (BMI) measured in $\mathrm{kg} / \mathrm{m}^{2}$ units. Participants classified as 
non-obese had a $\mathrm{BMI} \leq 29.99$, while participants classified as obese had a $\mathrm{BMI} \geq 30 .^{21}$ Hypertension was based on current antihypertensive treatment and/or having a blood pressure $(\mathrm{BP}) \geq 140 / 90 \mathrm{mmHg}$. Smoking status was ascertained based on participant's report. Participants were defined as non-smokers if they reported no smoking history or quit at least 10 years before the evaluation.

\section{Imaging}

All MR imaging were performed on the 3T system (Siemens, Erlangen, Germany). All participants underwent structural T-1 weighted, axial fluid attenuation inversion recovery (FLAIR), and susceptibility weighted imaging. FLAIR images were acquired with TR (repetition time) $=9000 \mathrm{~ms}$, TE (echo time) $=99 \mathrm{~ms}$ and TI (inversion time) $=2500 \mathrm{~ms}$; FA (flip angle) $130^{\circ}$, slice thickness: $3.3 \mathrm{~mm}$, FOV (field of view) $220 \mathrm{~mm}$, matrix $=256 \times 192$ reconstructed as $30256 \times 256$ images (See Figure 1).

WMH was graded from 0 to 3 on the Fazekas scale. ${ }^{22}$ Periventricular (PWMH) and deep white matter hyperintensities (DWMH) were graded separately and summed to create the total load (possible score 0-6). Severe WMH was defined as a total (summed) score $\geq 4$. We analyzed and calculated the following imaging markers:

1. Any DWMH (Fazekas 1-3), Any PWMH (Fazekas 1-3) and Any WMH Total (Fazekas score 1-6)

2. Severe DWMH (Score $\geq 2)$, Severe PWMH (Score $\geq 2$ ), and Severe WMH (Score $\geq 4$ )

\section{Data Analysis}

First, we performed frequency distribution and descriptive analyses (mean, standard deviation, and range) for target (white matter hyperintensities, obesity, and race/ethnicity) and confounding (age, sex, smoking status, and hypertension status) variables. Second, we performed chi-square analysis of $\mathrm{WMH}$ indicators and covariates to determine significant differences between African Americans and European Americans. Third, using the full sample, we performed logistic regression analysis investigating the independent and interactive effect of race/ethnicity and obesity on white matter hyperintensity load, adjusting for age, sex, hypertension, and smoking. Fourth, we performed stratified analyses to determine which factors predicted WMH differentially between European Americans and African Americans. Based on our hypothesis, we investigated whether the association between obesity and high white matter hyperintensity load differed by race/

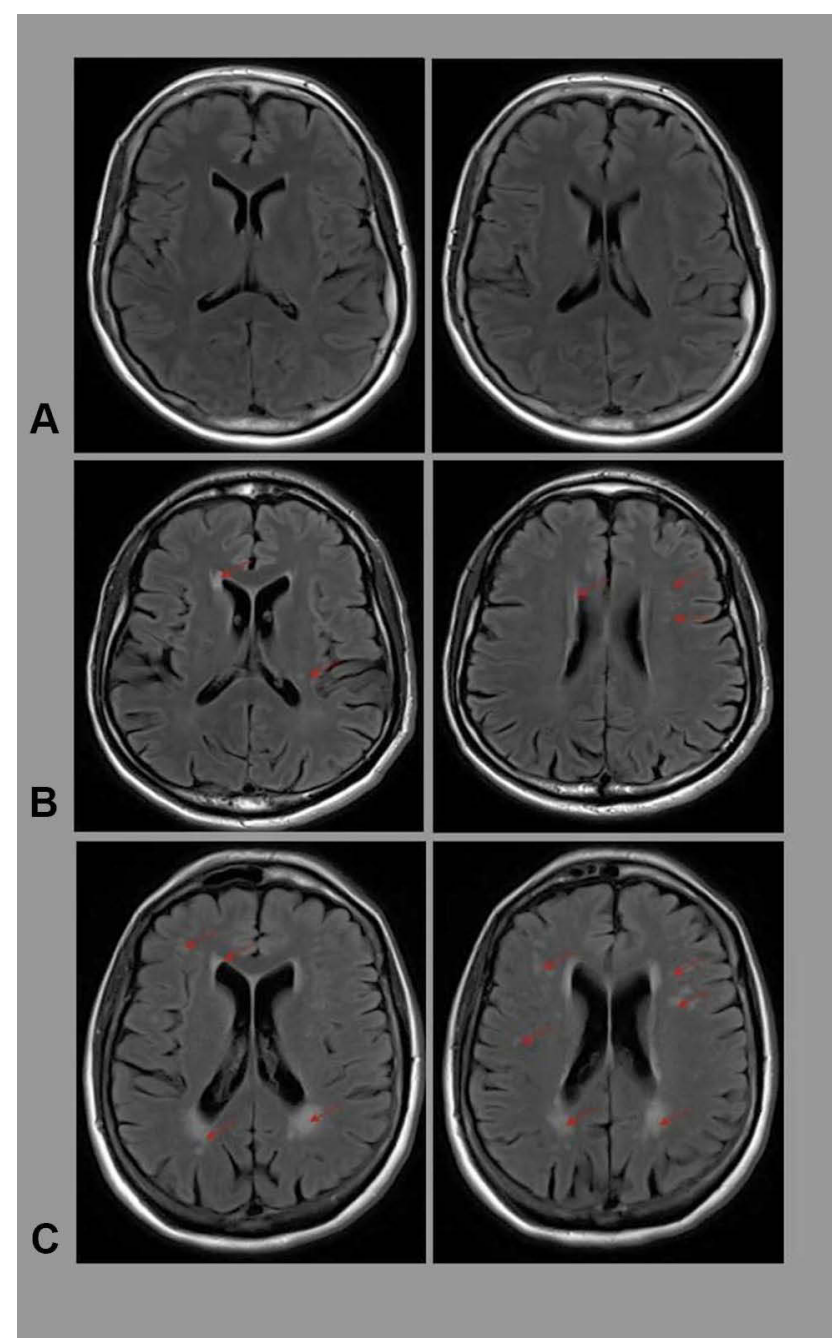

Figure I Examples of different severity of white matter lesions in study subjects. (A) None (65 year old European American female). (B) Thought present (66 year old African American male). (C) Pronounced (70 year old European American female).

ethnicity (African and European Americans) and by weight status (non-obese vs obese). Finally, since our sample had few African Americans compared to European Americans, we performed case controlled 1:1 matching of 24 African Americans and 24 European Americans. The two groups were matched based on age, sex, and education and had no significant differences in systolic blood pressure, hypertension, body mass index, obesity, and smoking history. We then repeated logistic regression analyses in the matched subgroup.

\section{Data Availability}

The dataset utilized for analyses of the current study are available from the corresponding author upon reasonable request. 


\section{Results}

Table 1 illustrates observed differences between African Americans and European Americans across several sociodemographic and clinical factors. European Americans were significantly older $(70.0 \pm 7.5$ yrs. vs $65.1 \pm 8.2$ yrs., $\mathrm{p}<0.05)$ and reported more years of formal education $(16.7 \pm 2.3$ yrs. vs $15.3 \pm 2.4$ yrs.) compared to African Americans (Table 1). Compared to European Americans, African Americans had poorer clinical outcomes with a greater percentage of obesity, mean body mass index, hypertension, systolic and diastolic blood pressure, and smoking history which was the only clinical factor statistically significant (Table 1). Although, African Americans had higher percentages of white matter hyperintensities in all categories compared to European Americans (Table 1), the differences did not reach statistical significance.

\section{Race Moderating the Relationship Between Obesity and White Matter Hyperintensity Load}

Regression analyses demonstrate that age and the race $\mathrm{x}$ obesity interaction variable significantly increased the likelihood of high white matter hyperintensity (WMH) load for the entire sample (Table 2). With every unit increase in age, the odds of high $\mathrm{WMH}$ load increased by $16 \%(\mathrm{OR}=1.16,95 \% \mathrm{CI}=1.09-1.23, \mathrm{p}<0.001)$. The interaction variable of race and obesity was significantly associated with high WMH load (OR=18.87, 95\% $\mathrm{CI}=1.17-303.58, \mathrm{p}<0.05)$. Race stratified analyses indicate that only age was independently associated with high WMH load for European Americans (Table 3). However, for African Americans, only obesity was

Table 2 Logistic Regression of Obesity, Race, and Obesity*Race on High WMH Load

\begin{tabular}{|l|l|l|l|l|}
\hline Variables & $\boldsymbol{\beta}$ & S.E. & OR (95\% C.I.) & Sig. \\
\hline Sex & 0.54 & 0.37 & $1.72(0.83-3.54)$ & 0.14 \\
Age & 0.15 & 0.03 & $1.16(1.09-1.23)$ & $<0.00 \mathrm{I}$ \\
Race & -0.64 & 1.12 & $0.53(0.20-6.5 \mathrm{I})$ & 0.56 \\
Obesity & -2.67 & 1.58 & $0.07(0.03-1.54)$ & 0.09 \\
Hypertension & -0.10 & 0.36 & $0.91(0.45-1.82)$ & 0.78 \\
Smoking & 0.75 & 0.66 & $2.12(0.58-7.77)$ & 0.26 \\
Race*Obesity & 2.94 & 1.42 & $18.87(1.17-303.58)$ & $<0.05$ \\
\hline
\end{tabular}

Notes: Logistic regression was performed in the subgroup of 292 (268 European Americans and 24 African Americans) who had all the clinical information available. Reference group: European Americans.

Abbreviation: $\mathrm{WMH}$, white matter hyperintensities. independently associated with high WMH load (Table 4). Obese African Americans were 27 times more likely to have a high WMH load compared to obese European Americans ( $\mathrm{OR}=27.65$, 95\% CI=1.47519.13, $\mathrm{p}<0.05)$. All regression analyses adjusted for hypertension and smoking history and neither of the two were significantly associated in any of our models (full and race stratified analyses).

Since the number of African Americans was small, we matched 1:1 all African American subjects $(n=24)$ with European American participants with respect to age, gender and education. In this matched subgroup, African Americans were more likely to have a higher WMH burden than European Americans $\left(27 \%\right.$ vs $4 \%, \mathrm{Chi}^{2}=5.3$, $\mathrm{p}=0.02$ ). We repeated logistic regression analyses in the matched subgroup with clinical data. The prevalence of hypertension, obesity and smoking were not different between the two groups ( $n=24$ AA and $n=24$ EA). Blood pressure (systolic and diastolic), and BMI were not different between the groups. The final binary logistic regression model (most parsimonious, and with highest $\mathrm{R}^{2}$ ) included only obesity status and race (marginally significant). Both obesity and being African American increased the risk of having high burden of WMH (Table 4). Result indicates that obesity significantly predicted WMH load for the matched sample.

\section{Obesity Moderating the Relationship Between Race and White Matter Hyperintensity Load}

Of the entire sample, only 66 individuals were classified as obese. Chi-square analysis of obese individuals indicates that $57 \%$ (4 out of 7) of obese African Americans had high white matter hyperintensity load, while 20\% (12 out of 59) of obese European Americans had high white matter load. Stratified analyses of obese and non-obese individuals indicate that sex and age predicted high white matter hyperintensity load among non-obese individuals (Table 5), while age, hypertension, and race predicted high white matter hyperintensity load among obese individuals (Table 5). These findings suggest that obesity moderates the relationship between race (specifically African Americans) and high white matter hyperintensity load.

\section{Discussion}

Results indicate that age and interaction between race and obesity were independently associated with high WMH load 
Table 3 Logistic Regression of Obesity and Race on White Matter Hyperintensity Load by Race

\begin{tabular}{|l|c|c|c|l|l|l|c|c|c|}
\hline \multicolumn{4}{|c|}{ European Americans } & \multicolumn{5}{c|}{ African Americans } \\
\hline Variables & $\boldsymbol{\beta}$ & S.E. & OR (95\% C.I.) & Sig. & Variables & $\boldsymbol{\beta}$ & S.E. & OR (95\% C.I.) & Sig. \\
\hline Sex & 0.53 & 0.38 & $1.71(0.83-3.54)$ & 0.16 & Sex & -1.05 & 2.18 & $0.35(0.01-25.16)$ & 0.63 \\
Age & 0.15 & 0.03 & $1.17(1.09-1.23)$ & $<0.001$ & Age & 0.03 & 0.17 & $1.04(0.82-1.30)$ & 0.77 \\
Obesity & 2.92 & 0.41 & $1.34(0.03-1.54)$ & 0.48 & Obesity & 3.32 & 1.50 & $27.66(1.47-519.13)$ & $<0.05$ \\
Hypertension & -0.19 & 0.37 & $0.82(0.45-1.82)$ & 0.78 & Hypertension & 1.43 & 1.71 & $4.16(0.14-119.79)$ & 0.40 \\
Smoking & 1.08 & 0.66 & $2.75(0.58-7.77)$ & 0.14 & Smoking & -19.54 & 16483.00 & $0.00(00-00)$ & 0.99 \\
\hline
\end{tabular}

Note: Logistic regressions were performed in the subgroup of 292 (268 European Americans and 24 African Americans) who had all the clinical information available.

Table 4 Logistic Regression of Obesity, Race, and on WMH Load in Matched Sample

\begin{tabular}{|l|l|l|l|l|}
\hline Variables & $\boldsymbol{\beta}$ & S.E. & OR (95\% C.I.) & Sig. \\
\hline Race & 1.99 & 1.20 & $7.36(0.71-76.7)$ & 0.09 \\
Obese & 2.03 & 0.99 & $7.64(1.08-53.8)$ & 0.04 \\
\hline
\end{tabular}

Notes: Logistic regression was performed in the matched subgroup of 48 participants (24 European Americans and 24 African Americans) who had all the clinical information available. Reference group: European American.

Abbreviation: $\mathrm{WMH}$, white matter hyperintensities.

in our full sample. Analyses stratified by race and weight status demonstrated that both race (specifically African Americans) and obesity modulated the risk of high white matter hyperintensity load. In race-stratified analyses, obesity predicted high WMH load only for African Americans, while only age predicted high WMH load among European Americans. In weight status stratified analyses, race, age, and hypertension predicted high WMH load for obese individuals, while sex and age predicted high WMH load for non-obese individuals. Matched sampling analyses indicate that obesity predicted high WMH load among African Americans and European Americans of the same age, sex, and education, with no significant differences in systolic blood pressure, hypertension, body mass index, obesity, and smoking history. Taken together, these findings indicate that obesity is a significant risk factor for high white matter hyperintensity load and that the obesogenic risk of WMH load is more pronounced in African Americans. In sum, the risk profile for high WMH load is different across racial groups (age-specific for European Americans and weightspecific for African Americans) and weight status (demographic-specific for non-obese individuals [age and sex] and demographic- and clinical-specific for obese individuals [age, race, and hypertension]).

Our findings fill a critical gap in our understanding of racial/ethnic differences in WMH load by positing that obesity (not age, hypertension, or smoking history) may explain the increased risk and burden of WMH among African Americans. Therefore, our findings have significant public health and clinical implications because they provide plausible mechanisms for intervention in racial/ ethnic differences in WMH between African Americans and European Americans. Although sampling limitations may equivocate our findings, we argue that obesity among African Americans significantly increases their risk for WMH. Although we interpret our findings cautiously, the fact that being African American alone did not increase an individual's risk for WMH suggests that the burden of WMH among African Americans may be reduced through lifestyle modification strategies (eg, weight loss management solutions) that promote healthy BMI, as demonstrated in the Look AHEAD study. ${ }^{18}$

Table 5 Logistic Regression of Obesity and Race on White Matter Hyperintensity Load by Obesity Status

\begin{tabular}{|l|c|c|c|c|l|c|c|c|c|}
\hline \multicolumn{4}{|c|}{ Non-Obese Individuals (n=266) } & \multicolumn{4}{c|}{ Obese Individuals (n=66) } \\
\hline Variables & $\boldsymbol{\beta}$ & S.E. & OR (95\% C.I.) & Sig. & Variables & $\boldsymbol{\beta}$ & S.E. & OR (95\% C.I.) & Sig. \\
\hline Sex & 0.96 & 0.45 & $2.6 I(I .09-6.27)$ & 0.03 & Sex & -1.24 & 0.93 & $0.29(0.05-1.79)$ & 0.18 \\
Age & 0.12 & 0.03 & $1.13(1.06-1.20)$ & $<0.001$ & Age & 0.43 & 0.14 & $1.54(1.17-2.04)$ & $<0.001$ \\
Hypertension & 0.44 & 0.42 & $1.56(0.68-3.56)$ & 0.30 & Hypertension & -2.61 & 1.02 & $0.07(0.01-0.54)$ & 0.01 \\
Smoking & 1.38 & 0.72 & $3.99(0.98-16.28)$ & 0.05 & Smoking & -20.70 & 1140.00 & $0.00(0.00-0.00)$ & 0.99 \\
Race & -0.74 & 1.08 & $0.48(0.06-3.98)$ & 0.50 & Race & 4.74 & 1.61 & $114.94(4.94-2674.06)$ & $<0.001$ \\
\hline
\end{tabular}

Notes: Logistic regressions were performed in subgroups of non-obese $(n=266 ; 209$ European Americans and 17 African Americans) and obese individuals ( $n=66 ; 59$ European Americans and 7 African Americans) who had all the clinical information available. 


\section{African Americans and Obesity}

Although race and obesity were not independently associated with WMH in the current study, the interaction of race and obesity was associated with WMH. This highlights that there is something unique in the interaction of race (being African American) and obesity on WMH. Epidemiological data highlight a significant racial/ethnic difference across obesity between European Americans and African Americans, with African Americans twenty years or older having a higher prevalence of Grade 1 $\left(\mathrm{BMI} \geq 30 \quad \mathrm{~kg} / \mathrm{m}^{2} ; \quad 44.1 \% \quad\right.$ vs $32.8 \%$ ), Grade 2 (BMI $\geq 35 \mathrm{~kg} / \mathrm{m}^{2} ; 21.9 \%$ vs $13.6 \%$ ), and Grade 3 obesity $\left(\mathrm{BMI} \geq 40 \mathrm{~kg} / \mathrm{m}^{2} ; 11.1 \%\right.$ vs $\left.5.2 \%\right) .{ }^{23}$ More recent evidence indicates the prevalence of being overweight and obese among African Americans is growing. ${ }^{24}$ As a result, African Americans are at increased risk for obesity-related morbidity and mortality, such as CVD, and small vessel disease like WMH as demonstrated in our study. ${ }^{25}$

The deleterious effect of obesity on CVD may be proximal, distal, or insidious. Proximally, obesity may lead to myocardial infarction, stroke, and diabetes, which are more prevalent among African Americans, relative to other racial/ethnic groups. ${ }^{26}$ Distally, obesity increases the risk of small vessel disease (marked by WMH) which may manifest beyond a CVD event and may lead to neurodegeneration, vascular cognitive impairment, and dementia. ${ }^{27}$ Obesity also has an insidious effect, whereby obesity over the life-course may lead to an accumulation of age-related WMH, leukoaraiosis, and therefore may lead to elevated risk of mild cognitive impairment, dementia, depression, falls, gait disturbance, and stroke later in life. ${ }^{28}$ Our findings suggest that obese African Americans are more susceptible to $\mathrm{WMH}$, independent of the usual risk factors of WMH. Although, hypertension and smoking history were not significant in most of our models (except for significantly predicting high WMH load among obese individuals), it is likely that this is due to small sample size. It is also likely that hypertensive African Americans in our study have earlier ages of onset, longer treatment histories, and greater levels of controlled blood pressure for long periods of time, protecting them from developing WMH. ${ }^{29}$ However, the lack of evidence to support smoking history as a significant predictor of WMH is consistent with previous studies and may be attributed to lower smoking intensity among African Americans and their preponderance of using menthol cigarettes. ${ }^{30}$ An additional explanation for our finding may be that obesity compared to high blood pressure was a stronger predictor of high WMH load in our sample. Our conjecture is buttressed by evidence that hypertension was only predictive of high WMH load in obese individuals in our sample.

\section{African Americans, Obesity, Increased CVD Risk and Cerebral Small Vessel Disease}

Our study is not the first to suggest that obesity independently increases CVD risk, ${ }^{31}$ or that obesity may explain racial/ethnic differences in CVD between African Americans and European Americans. ${ }^{25}$ However, our finding that obesity may contribute to differential estimates of high WMH burden between African Americans and European Americans is novel. Since WMH explain a significant proportion of the variance in dementia and CVD (eg, stroke) estimates, ${ }^{32,33}$ preventing and reducing WMH may lower the disproportionate prevalence of CVD among African Americans. ${ }^{34}$ Additionally, since preventive strategies like weight loss through exercise and diet compared to support and education have successfully reduced WMH risk and improved overall brain function among diabetics, ${ }^{18}$ we argue that such lifestyle and behavioral preventive strategies may reduce WMH-related morbidity and mortality among racial/ethnic minority groups, particularly African Americans. The confluence of cardiovascular risk factors (obesity and hypertension) and WMH load may result in diminished brain activity, such as reduced glucose uptake in regions of the brain with hyperintensities and interconnected gray matter, and thus increase an individual's risk for abnormalities in brain function and neurodegenerative disease. ${ }^{35,36}$

\section{Risk of White Matter Hyperintensities Among Non-Obese Individuals}

Although we found that obesity was a driver for WMH, our finding that sex and age were strong predictors of WMH among non-obese individuals highlights other risk phenotypes. As indicated above, age is a traditional risk factor, and mounting evidence suggest that sex, specifically women are at increased risk for vascular disease and small vessel diseases like, hypertension, stroke, and diabetes. ${ }^{37,38}$ In one epidemiological study, women compared to men, specifically, non-Latino white women in the NYC NHANES dataset had the greatest cardiovascular disease risk. ${ }^{39}$ However, unique sex and race risk patterns 
were found where Non-Latino black women were more at risk for being overweight/obese, hypertensive, hypercholesteroemic, and diabetic, compared to their race and sex counterparts. Based on these findings it is therefore likely that non-obese females may be more at risk for $\mathrm{WMH}$ because of other clinical risk factors and thus should be stratified differently in clinical settings.

\section{Limitations}

Our findings are interpreted with caution due to possible design, sampling, and methodological limitations. One potential limitation is the use of BMI as a proxy for obesity and risk factor of severe WMH. Body mass index may not be an accurate measurement of determining body fat difference between African Americans and European Americans, as it is well documented that African Americans generally have higher lean mass and lower fat mass. ${ }^{40-42}$ African Americans may have a higher body mass index because they generally have more muscle mass as opposed to fat mass, which is linked with poor cardiovascular and cerebrovascular health. ${ }^{43-45}$ Future studies should investigate the insidious effects of obesity on white matter integrity over time. ${ }^{46}$ Our use of a visual rating scale, the Fazekas scale, is limiting because it captures a crude rather than granular measure of $\mathrm{WMH}$. Lastly, the low percentage of African Americans in our sample precludes making generalizable claims of the effects obesity has on WMH load across racial/ethnic groups.

Future studies should investigate whether the pathophysiology of severe WMH determines the disease course and if differential pathways leading to $\mathrm{WMH}$ (eg, hypertension, smoking, and obesity) determine the type of disease developed (eg, cerebrovascular disease, stroke, mild cognitive impairment, and/or dementia). For example, if an individual's WMH is caused by obesity are they more likely to develop dementia or cerebrovascular disease? Additionally, given that research indicates minor neuropsychological alterations in individuals with small vessel disease without cognitive impairments, future studies should include a line of research that explores whether there are differences in cognitive alterations if an individual's WMH is caused by obesity. ${ }^{47}$ Second, given the posited relationship between obesity and WHM overall, future studies should explore specific underpinnings that would explicate the findings of obesity's influence on WMHs in African Americans. For example, examinations of the biopsychosocial and environmental factors that influence racial/ethnic differences and how they relate to these findings regarding obesity and WMH. Third, future studies should investigate whether there is a genetic explanation for the impact obesity and race have on severe $\mathrm{WMH}$ among African Americans and European Americans. Based on previous findings among Mexican-Americans, obesity (BMI and waist circumference) was negatively associated with white matter integrityfractional anisotropy (whole brain and regional measurement of cerebral white matter integrity). ${ }^{48}$ In previous studies, shared genetic variance between obesity and regions of the brain, such as the genu, body, and selenium of corpus callosum, internal capsule, thalamic radiation, and in the superior fronto-occipital fasciculus, were significant, which suggests there may be a significant genetic underpinning to WMH. Finally, future studies should investigate whether weight loss reduces prevalence and severity of small vessel diseases, marked by $\mathrm{WMH}$, and the long-term effects of obesity on WMH.

\section{Conclusion}

Extant research indicates that African Americans compared to European Americans are at increased risk for WMH, a marker of small vessel brain disease and a significant predictor of CVD, mild cognitive impairment, and dementia. However, it is unclear why African Americans have a higher risk of $\mathrm{WMH}$, especially given that race is a social construct, and previous studies indicate that smoking and hypertension do not explain the racial/ethnic difference in WMH. Our findings indicate that obesity may explain differential estimates of WMH between African Americans and European Americans. These findings are sobering and encouraging. Sobering because obesity, a growing and pervasive health risk condition in the United States especially among African Americans (who are twice as likely to be to be obese), is responsible for the differential burden of white matter hyperintensity load. Encouraging because these results, albeit preliminary, indicate the possibility of preventing or reducing WMH risk through obesity prevention and management, or weight loss. Future studies should investigate the longitudinal effects of obesity on WMH load differentially across races.

\section{Data Sharing Statement}

The dataset utilized for analyses of the current study are available from the corresponding author upon reasonable request. 


\section{Statement of Ethics}

All procedures performed in this study involving human participants were completed in accordance with the ethical standards of the NYU Langone Health Institutional Review Board and with the Helsinki declaration and its amendments.

\section{Acknowledgments}

The authors acknowledge the support of several funding agencies and efforts of study staff, key personnel, and participants who all contributed to make the study successful. All coauthors meet the criteria for authorship, including acceptance of responsibility for the scientific content of the paper. They have seen and agreed on the contents of the paper and there is no financial conflict or conflicts of interests to report. They certify that the submission is the original work and is not under review at any other publication.

\section{Author Contributions}

All authors made a significant contribution to the work reported, whether that is in the conception, study design, execution, acquisition of data, analysis and interpretation, or in all these areas; took part in drafting, revising or critically reviewing the article; gave final approval of the version to be published; have agreed on the journal to which the article has been submitted; and agree to be accountable for all aspects of the work.

\section{Funding}

This research was supported by funding from the National Institutes of Health: K01HL135452, K07AG052685, R01HL152453, R01MD007716, R01HL142066, R01AG0 67523, R01AG056031, R01NS104364, MdeL (RF1AG 057570, R56 AG058913, R01 AG012101, R01 AG02 2374, R01 AG013616), R01 HL111724, R01AG05653, R01AG056031, and R25HL105444. The funding sources had no role in the design, conduct, or analysis of the study, or in the decision to submit the manuscript for publication.

\section{Disclosure}

The authors have no conflicts of interest to declare.

\section{References}

1. Ferri CP, Prince M, Brayne C, et al. Global prevalence of dementia: a Delphi consensus study. Lancet. 2005;366(9503):2112-2117. doi:10. 1016/S0140-6736(05)67889-0

2. Tang MX, Cross $P$, Andrews $H$, et al. Incidence of $A D$ in African-Americans, Caribbean Hispanics, and Caucasians in northern Manhattan. Neurology. 2001;56(1):49-56. doi:10.1212/WNL.56.1.49
3. Miles TP, Froehlich TE, Bogardus ST, Inouye SK. Dementia and race: are there differences between African Americans and Caucasians? J. Am Geriatr Soc. 2001;49(4):477-484. doi:10.1046/ j.1532-5415.2001.49096.x

4. Agyemang C, Van De Vorst IE, Koek HL, et al. Ethnic variations in prognosis of patients with dementia: a prospective nationwide registry linkage study in the Netherlands. J Alzheimer's Dis. 2017;56(1).

5. Kernan WN, Ovbiagele B, Black HR, et al. Guidelines for the prevention of stroke in patients with stroke and transient ischemic attack: a guideline for healthcare professionals from the American Heart Association/American Stroke Association. Stroke. 2014;45 (7):2160-2236. doi:10.1161/STR.0000000000000024

6. Meschia JF, Bushnell C, Boden-Albala B, et al. Guidelines for the primary prevention of stroke: a statement for healthcare professionals from the American heart association/American stroke association. Stroke. 2014;45(12):3754-3832.

7. Arboix A, Blanco-Rojas L, Martí-Vilalta JL. Advancements in understanding the mechanisms of symptomatic lacunar ischemic stroke: translation of knowledge to prevention strategies. Expert Rev Neurother. 2014;14(3):261-276. doi:10.1586/14737175.2014.884926

8. George AE, de Leon MJ, Kalnin A, Rosner L, Goodgold A, Chase N. Leukoencephalopathy in normal and pathologic aging: 2. MRI of brain lucencies. Am J Neuroradiol. 1986;7(4):567-570.

9. George AE, de Leon MJ, Gentes CI, et al. Leukoencephalopathy in normal and pathologic aging: 1. CT of brain lucencies. Am J Neuroradiol. 1986;7(4):561-566.

10. Debette S, Markus HS. The clinical importance of white matter hyperintensities on brain magnetic resonance imaging: systematic review and meta-analysis. BMJ. 2010;341(7767):288. doi:10.1136/ bmj.c3666

11. Liao D, Cooper L, Cai J, et al. The prevalence and severity of white matter lesions, their relationship with age, ethnicity, gender, and cardiovascular disease risk factors: the ARIC study. Neuroepidemiology. 1997;16(3):149-162. doi:10.1159/000368814

12. Nyquist PA, Bilgel MS, Gottesman R, et al. Extreme deep white matter hyperintensity volumes are associated with African American race. Cerebrovasc Dis. 2014;37(4):244-250. doi:10.1159/000358117

13. Gottesman RF, Coresh J, Catellier DJ, et al. Blood pressure and white-matter disease progression in a biethnic cohort: atherosclerosis risk in communities (ARIC) study. Stroke. 2010;41(1):3-8. doi:10.1161/STROKEAHA.109.566992

14. Manolio TA, Kronmal RA, Burke GL, et al. Magnetic resonance abnormalities and cardiovascular disease in older adults the cardiovascular health study. Stroke. 1994;25(2):318-327. doi:10.1161/01. STR.25.2.318

15. Habes M, Erus G, Toledo JB, et al. Regional tract-specific white matter hyperintensities are associated with patterns to aging-related brain atrophy via vascular risk factors, but also independently. Alzheimer's Dement Diagnosis, Assess Dis Monit. 2018;10:278-284.

16. Gorelick PB. Cerebrovascular disease in African Americans. Stroke. 1998;29(12):2656-2664. doi:10.1161/01.STR.29.12.2656

17. White H, Boden-Albala B, Wang C, et al. Ischemic stroke subtype incidence among whites, blacks, and Hispanics: the northern Manhattan study. Circulation. 2005;111(10):1327-1331. doi:10.1161/01.CIR.0000157736.19739.D0

18. Espeland MA, Erickson K, Neiberg RH, et al. Brain and white matter hyperintensity volumes after 10 years of random assignment to lifestyle intervention. Diabetes Care. 2016;39(5):764-771. doi:10.2337/dc152230

19. Bech P, Kastrup M, Rafaelsen OJ. Mini-compendium of rating scales for states of anxiety depression mania schizophrenia with corresponding DSM-III syndromes. Acta Psychiatr Scand Suppl. 1986;326:1-37.

20. Folstein MF, Folstein SE, McHugh PR. Mini-mental state". A practical method for grading the cognitive state of patients for the clinician. J Psychiatr Res. 1975;12(3):189-198. doi:10.1016/ 0022-3956(75)90026-6 
21. Donato KA. Executive summary of the clinical guidelines on the identification, evaluation, and treatment of overweight and obesity in adults. Arch Intern Med. 1998;158(17):1855-1867.

22. Fazekas F, Chawluk JB, Alavi A, Hurtig HI, Zimmerman RA. MR signal abnormalities at $1.5 \mathrm{~T}$ in Alzheimer's's dementia and normal aging. Am J Roentgenol. 1987;149(2):351-356. doi:10.2214/ajr.149.2.351

23. Flegal KM, Shepherd JA, Looker AC, et al. Comparisons of percentage body fat, body mass index, waist circumference, and waist-stature ratio in adults. Am J Clin Nutr. 2009;89(2):500-508. doi:10.3945/ajen.2008.26847

24. Mozaffarian D, Benjamin EJ, Go AS, et al. Executive summary: heart disease and stroke statistics-2016 update: a Report from the American Heart Association. Circulation. 2016. doi/10.1161/ CIR.0000000000000366

25. Brandon LJ, Proctor L, Cole CL. Influence of obesity assessments on cardiometabolic risks in African and European American women. Ethn Dis. 2014;24(4):475-480.

26. Frieden TR, Houry D, Baldwin G. Traumatic brain injury in the United States: epidemiology and rehabilitation. CDC NIH Rep Congr. 2015. Available from: https://www.cdc.gov/traumaticbraininjury/pdf/tbi report to congress epi and rehab-a.pdf.

27. Pasha EP, Birdsill AC, Parker P, Elmenshaway A, Tanaka H, Haley AP. Visceral adiposity predicts subclinical white matter hyperintensities in middle-aged adults. Med Sci Sport Exerc. 2016;48:426. doi:10.1249/01.mss.0000486282.41474.0a

28. Miki Y, Sakamoto S. Age-related white matter lesions (leukoaraiosis): an update. Brain and Nerve. 2013;65(7):789-799.

29. Justin Thomas S, Booth JN, Dai C, et al. Cumulative incidence of hypertension by 55 years of age in blacks and whites: the cardia study. J Am Heart Assoc. 2018;7(14).

30. Power MC, Deal JA, Sharrett AR, et al. Smoking and white matter hyperintensity progression: the ARIC-MRI Study. Neurology. 2015;84(8):841-848. doi:10.1212/WNL.0000000000001283

31. Rashid MN, Fuentes F, Touchon RC, Wehner PS. Obesity and the risk for cardiovascular disease. Prev Cardiol. 2003;6(1):42-47. doi:10.1111/j.1520-037X.2003.01358.x

32. Rost NS, Rahman RM, Biffi A, et al. White matter hyperintensity volume is increased in small vessel stroke subtypes. Neurology. 2010;75(19):1670-1677. doi:10.1212/WNL.0b013e3181fc279a

33. Smith EE, Egorova S, Blacker D, et al. Magnetic resonance imaging white matter hyperintensities and brain volume in the prediction of mild cognitive impairment and dementia. Arch Neurol. 2008;65 (1):94-100. doi:10.1001/archneurol.2007.23

34. Modir R, Gardener H, Wright CB. Blood pressure and white matter hyperintensity volume - a review of the relationship and implications for stroke prediction and prevention. Eur Neurol Rev. 2012;7 (3):174-177. doi:10.17925/ENR.2012.07.03.174

35. Glodzik L, Rusinek H, Li J, et al. Reduced retention of Pittsburgh compound B in white matter lesions. Eur J Nucl Med Mol Imaging. 2015;42(1):97-102. doi:10.1007/s00259-014-2897-1
36. Glodzik L, Kuceyeski A, Rusinek H, et al. Reduced glucose uptake and A $\beta$ in brain regions with hyperintensities in connected white matter. Neuroimage. 2014;100:684-691. doi:10.1016/j.neuroimage.2014.06.060

37. Ahmadi-Ahangar A, Sheikh Z, Khani A, Taheri ST. Sex-differences in the incidence of stroke: should we change our traditional belief?. Clin Neurol Neurosurg. 2015;131:77. doi:10.1016/j.clineuro.2015.01.031

38. Reeves MJ, Bushnell CD, Howard G, et al. Sex differences in stroke: epidemiology, clinical presentation, medical care, and outcomes. Lancet Neurol. 2008;7(10):915-926. doi:10.1016/S1474-4422(08)70193-5

39. Kanchi R, Perlman SE, Chernov C, et al. Gender and race disparities in cardiovascular disease risk factors among New York City Adults: New York City health and nutrition examination survey (NYC HANES) 2013-2014. J Urban Heal. 2018;95(6):801-812. doi:10.1007/s11524-018-0287-x

40. Gillespie SL, Christian LM. Body mass index as a measure of obesity: racial differences in predictive value for health parameters during pregnancy. J Women's Heal. 2016;25(12):1210-1218. doi:10.1089/jwh.2016.5761

41. Carroll JF, Chiapa AL, Rodriquez M, et al. Visceral fat, waist circumference, and BMI: impact of race/ethnicity. Obesity. 2008;16 (3):600-607. doi:10.1038/oby.2007.92

42. Bergman RN, Stefanovski D, Buchanan TA, et al. A better index of body adiposity. Obesity. 2011;19(5):1083-1089. doi:10.1038/oby.2011.38

43. Aloia JF, Vaswani A, Mikhail M, Flaster ER. Body composition by dual-energy X-ray absorptiometry in black compared with white women. Osteoporos Int. 1999;10(2):114-119. doi:10.1007/s001980 050204

44. Fernández JR, Heo M, Heymsfield SB, et al. Is percentage body fat differentially related to body mass index in Hispanic Americans, African Americans, and European Americans? Am J Clin Nutr. 2003;77(1):71-75. doi:10.1093/ajcn/77.1.71

45. Rahman M, Temple JR, Breitkopf CR, Berenson AB. Racial differences in body fat distribution among reproductive-aged women. Metabolism. 2009;58(9):1329-1337. doi:10.1016/j.metabol.2009.04. 017

46. De Groot M, Verhaaren BFJ, De Boer R, et al. Changes in normal-appearing white matter precede development of white matter lesions. Stroke. 2013;44(4):1037-1042. doi:10.1161/STROKEAHA. 112.680223

47. Blanco-Rojas L, Arboix A, Canovas D, Grau-Olivares M, Oliva Morera JC, Parra O. Cognitive profile in patients with a first-ever lacunar infarct with and without silent lacunes: a comparative study. BMC Neurol. 2013;13(1):1-7. doi:10.1186/1471-2377-13-203

48. Spieker EA, Kochunov P, Rowland LM, et al. Shared genetic variance between obesity and white matter integrity in Mexican Americans. Front Genet. 2015;5.
Clinical Interventions in Aging

\section{Publish your work in this journal}

Clinical Interventions in Aging is an international, peer-reviewed journal focusing on evidence-based reports on the value or lack thereof of treatments intended to prevent or delay the onset of maladaptive correlates of aging in human beings. This journal is indexed on PubMed Central, MedLine, CAS, Scopus and the Elsevier
Bibliographic databases. The manuscript management system is completely online and includes a very quick and fair peer-review system, which is all easy to use. Visit http://www.dovepress.com/ testimonials.php to read real quotes from published authors. 